\title{
A new sample of extremely/ultra metal-poor stars
}

\author{
A E García Pérez ${ }^{1}$, N Christlieb ${ }^{2}$, S G Ryan ${ }^{1}$, T C Beers ${ }^{3}$, W Aoki ${ }^{4}$, \\ M Asplund ${ }^{5}$, P S Barklem ${ }^{6}$, M S Bessell ${ }^{7}$, K Eriksson ${ }^{6}$, A Frebel $^{8}$, \\ B Gustafsson ${ }^{6}$, A J Korn ${ }^{6}$, B Nordström ${ }^{9}$ and J E Norris ${ }^{7}$ \\ ${ }^{1}$ Department of Physics, Astronomy and Mathematics, University of Hertfordshire, College Lane, \\ Hatfield AL10 9AB, UK \\ ${ }^{2}$ Center for Astronomy, Landessternwarte, Königstuhl 12, University of Heidelberg, Heidelberg 69177, \\ Germany \\ ${ }^{3}$ Department of Physics and Astronomy and JINA (Joint Institute for Nuclear Astrophysics), \\ Michigan State University, East Lansing, MI 48824, USA \\ ${ }^{4}$ National Astronomical Observatory of Japan, 2-21-1 Osawa, Mitaka, Tokyo 181-8588, Japan \\ ${ }^{5}$ Max-Planck Institute for Astrophysics, Karl-Schwarzschild-Strasse 1, Postfach 1317, \\ Garching b. München 8574, Germany \\ ${ }^{6}$ Department of Physics and Astronomy, Uppsala University, Box 515, Uppsala 75120, Sweden \\ ${ }^{7}$ Research School of Astronomy and Astrophysics, The Australian National University, \\ Mount Stromlo Observatory, Cotter Road, Weston, ACT 2611, Australia \\ ${ }^{8}$ McDonald Observatory, University of Texas, Austin, TX 78712-0259, USA \\ ${ }^{9}$ Niels Bohr Institute, Copenhagen University, Juliane Maries Vej 30, Copenhagen 2100, Denmark \\ E-mail: a.e.garcia-perez@herts.ac.uk
}

Received 17 September 2008

Accepted for publication 22 September 2008

Published 19 December 2008

Online at stacks.iop.org/PhysScr/T133/014036

\begin{abstract}
A sample of 30 very metal-poor stars from the Hamburg-European Southern Observatory (ESO) objective-prism survey have been observed at high spectral resolution at the Very Large Telescope (VLT) using the Ultraviolet and Visual Echelle Spectrograph (UVES). Two of the observed stars are very interesting not only because of their very low iron content, approximately four orders of magnitude lower than the solar value, but also because we detected the neutral lithium resonance line at $670.8 \mathrm{~nm}$. Hydrogen lines suggest that the two observed stars have effective temperatures around 6000-6250 K and according to isochrones, they are either on the main-sequence or on the subgiant branch, in which case they would probably be the most metal-poor dwarfs or warm subgiants with lithium detections known. These detections would allow to determine more accurately the slope of the trend of the lithium abundance with $[\mathrm{Fe} / \mathrm{H}]$ than was possible with samples of unevolved stars restricted to higher metallicities.
\end{abstract}

PACS numbers: 97.10.Ex, 97.10.Tk, 97.20.Tr, 97.20.Wt

\section{Introduction}

The first stars in the Galaxy are believed to had been very massive and had short lifetimes so they are not observable nowadays. However, they have left traces of their nucleosynthesis processes in the atmospheres of second generation stars. The study of the chemical composition of extremely $(-4.00 \leqslant[\mathrm{Fe} / \mathrm{H}] \leqslant-3.00)$ and ultra $(-5.00 \leqslant[\mathrm{Fe} / \mathrm{H}] \leqslant-4.00)$ metal-poor stars are indirectly revealing the history of the first stars in the Galaxy.

In the Hamburg/ESO objective-prism survey (HES; Christlieb et al 2008), three stars with an iron abundance lower than $10^{-4}$ times the solar value have been discovered: HE 1327-2326 $([\mathrm{Fe} / \mathrm{H}] \sim-5.5$; Frebel et al 2005), HE 0107-5240 $([\mathrm{Fe} / \mathrm{H}] \sim-5.2$; Christlieb et al 2002) and $\mathrm{HE}$ 0557-4840 $([\mathrm{Fe} / \mathrm{H}] \sim-4.8 ;$ Norris et al 2007). According 
to Beers and Christlieb (2005), the probability of finding these ultra metal-poor stars is very low. Until the recent discovery of HE $0557-4840$, it was thought that there is a 'gap' in the metallicity range $-5.00 \leqslant[\mathrm{Fe} / \mathrm{H}] \leqslant-4.00$. A physical mechanism associated with the formation of $\mathrm{H}_{2}$ in star-forming cores (Tsuribe and Omukai 2008) has been proposed to explain the gap; another explanation arises from stochastic models of Galactic chemical evolution (Karlsson 2006). The discovery of more stars at $[\mathrm{Fe} / \mathrm{H}]<-4.0$ would provide insights on the physics of fragmentation of star-forming cores and the chemical evolution.

The history of the discovery of HE 1327-2326 and HE 0107-5240 has shown that $[\mathrm{Fe} / \mathrm{H}]$ can be overestimated when estimated from moderate-resolution (i.e. $\Delta \lambda=2 \AA$ ) follow-up spectroscopy of metal-poor candidates selected in the survey, e.g. due to the presence of interstellar Ca II $\mathrm{K}$ absorption, blending with $\mathrm{CH}$ lines, or a $\mathrm{Ca}$ abundance larger than that assumed in the $[\mathrm{Fe} / \mathrm{H}]$ calibration. We have therefore started an effort aiming at the identification of more stars at $[\mathrm{Fe} / \mathrm{H}]<-4.0$ by obtaining high-resolution spectra of stars from the HES for which moderate resolution (i.e. $\Delta \lambda=2 \AA$ ) indicates that they have $[\mathrm{Fe} / \mathrm{H}]<-3.5$. Thirty such stars were successfully observed at the $8-\mathrm{m}$ Very Large Telescope (VLT) with the Ultraviolet and Visual Echelle Spectrograph (UVES).

In this paper, we present preliminary results for stars which are of particular interest due to their low iron abundance, and because we have detected lithium in their spectra. Furthermore, one of them may be in the $-5.00 \leqslant$ $[\mathrm{Fe} / \mathrm{H}] \leqslant-4.00$ gap zone. The results of a thorough abundance analysis of the full sample will be presented elsewhere.

\section{Sample of candidates}

Spectra covering blue and red wavelengths at resolving powers $(\lambda / \delta \lambda) 40000-60000$ were acquired simultaneously using UVES in dichroic mode. In the first step of the Visitor Mode observations, spectra with a signal-to-noise ratio of $S / N \sim 30$ per pixel at $400 \mathrm{~nm}$ were obtained. A quick-look analysis of the spectra was performed at the telescope, and for stars that were confirmed to have $[\mathrm{Fe} / \mathrm{H}]<-3.5$, or which were otherwise interesting, the exposure time was extended to reach $S / N=80$ for the blue arm spectra and 150 for the red arm spectra. We found that the spectra of many of the observed stars showed strong $\mathrm{G}$ bands of $\mathrm{CH}$ (near $432.2 \mathrm{~nm}$ ). The Fe I lines of four stars were very weak suggesting a very low iron content, around $10^{-4}$ the solar iron content, whereas two of these (HE 0233-0343 and HE 0945-1435) seem to be extremely interesting because of detections of their lithium line at $670.8 \mathrm{~nm}$. We will seek to confirm the results using a more thorough analysis based on a proper data reduction which we have completed recently. This analysis is being carried out, and here we present some of the preliminary results for HE 0233-0343 and HE 0945-1435.

\section{Iron content}

The stellar parameters of the two stars are summarized in table 1. They were determined as follows. Our $T_{\text {eff }}$
Table 1. Stellar parameters and lithium abundance of two HES stars for two possible surface gravities.

\begin{tabular}{llcccc}
\hline Star & $T_{\text {eff }}(\mathrm{K})$ & $\log g(\mathrm{cgs})$ & {$[\mathrm{Fe} / \mathrm{H}]$} & $\xi_{\mathrm{t}}\left(\mathrm{km} \mathrm{s}^{-1}\right)$ & $A(\mathrm{Li})$ \\
\hline HE 0233-0343 & 6250 & 3.5 & -4.20 & 1.5 & 1.90 \\
& 6000 & 4.5 & -4.45 & 1.5 & 1.70 \\
HE 0945-1435 & 6250 & 3.5 & -3.80 & 1.5 & 1.99 \\
& 6000 & 4.5 & -4.09 & 1.5 & 1.80 \\
\hline
\end{tabular}

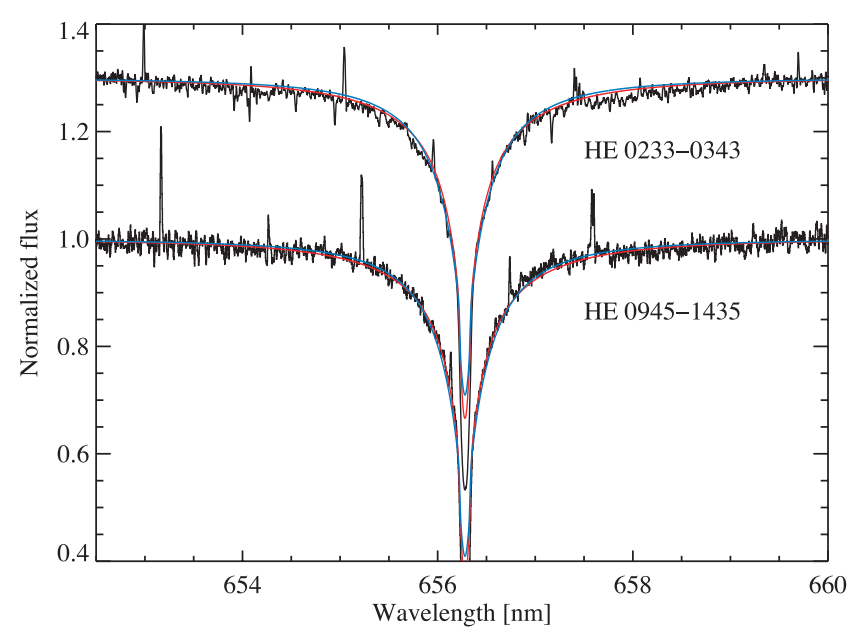

Figure 1. The $\mathrm{H} \alpha$ observed spectra of HE 0233-0343 and HE 0945-1435 offset vertically for clarity. Spectral synthesis for two different $T_{\text {eff }} / \log g /[\mathrm{Fe} / \mathrm{H}]$ value sets are shown: in blue $6000 / 4.5 /-4.00$ and in red $6250 / 4.5 /-4.00$.

estimates are based on a profile analysis of the observed $\mathrm{H} \alpha$ line (see figure 1) following the procedure in Barklem et al (2002). The other stellar parameter required for the abundance determinations is the surface gravity which was estimated from the Yonsei-Yale isochrone of $[\mathrm{Fe} / \mathrm{H}]=-3.29$ and an age of $12 \mathrm{Gyr}$ (Demarque et al 2004). Since an accurate spectroscopic determination of the surface gravity $\log g$ is not yet available, the evolutionary state of the stars is currently unclear. Stars are either on the main sequence $(\log g=4.5)$ or on the warm subgiant branch $(\log g=3.5)$. The microturbulence was set to $\xi_{\mathrm{t}}=1.5 \mathrm{~km} \mathrm{~s}^{-1}$.

The Fe I lines of the HES stars with lithium detections are much weaker than the lines of the more metal-rich star HD $140283([\mathrm{Fe} / \mathrm{H}] \sim-2.40)$, as observed in figure 2, where the observed spectra of two Fe I lines in the three stars are compared. Iron abundances were determined by comparing the measured equivalent widths of a set of eight $\mathrm{Fe}$ I lines in the wavelength range $381.0-406.5 \mathrm{~nm}$ with synthesized lines. The spectral synthesis was carried out with the Uppsala suite of codes and the new MARCs model atmospheres (Gustafsson et al 2008). The analysis of the $\mathrm{Fe} \mathrm{I}$ lines suggests $[\mathrm{Fe} / \mathrm{H}] \sim-4.20 /-4.45$ and $[\mathrm{Fe} / \mathrm{H}] \sim-3.80 /-4.09$ for HE 0233-0343 and HE 0945-1435, respectively, for the two gravities. These results are expected to be affected by departures from local thermodynamic equilibrium (LTE) and should be corrected of non-local thermodynamic equilibrium (NLTE) effects (Collet et al 2005).

The stellar parameter determination needs further refinement, in particular the surface gravity. However, the range of stellar parameters assumed in this work is wide 


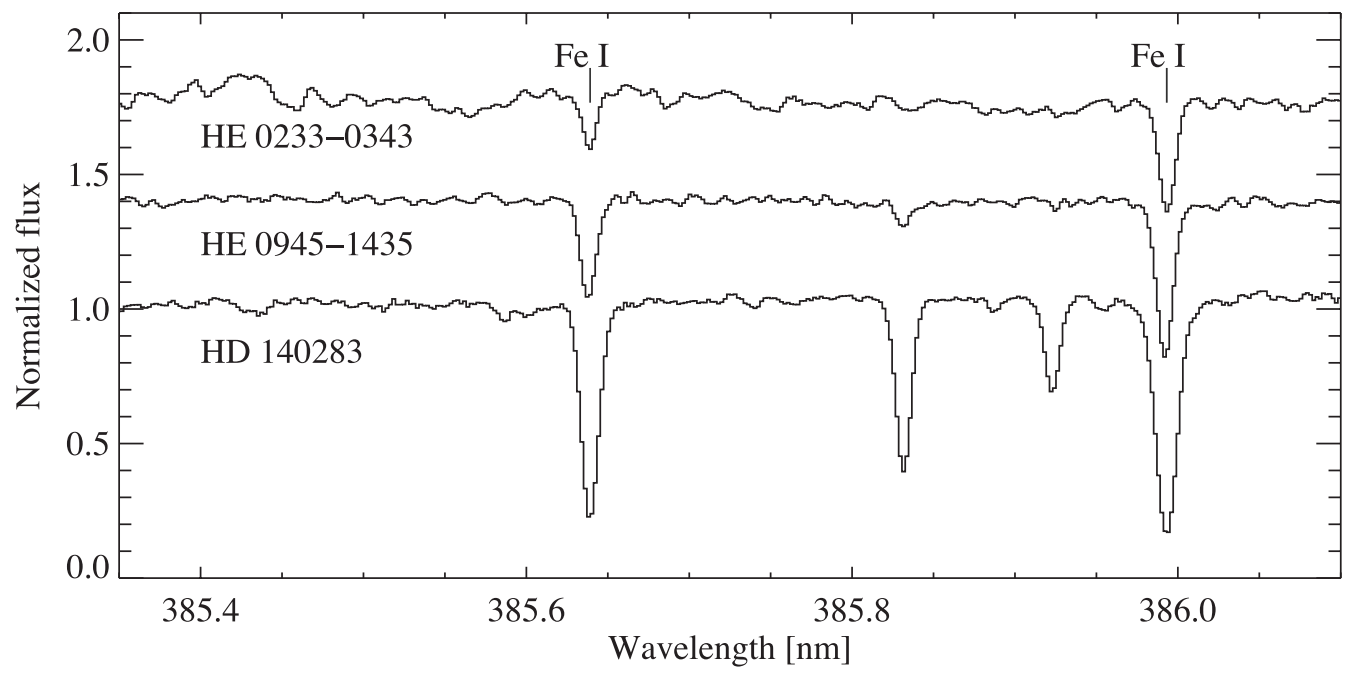

Figure 2. VLT/UVES spectra of the Fe I lines at $385.63 \mathrm{~nm}$ and $385.99 \mathrm{~nm}$ for HE 0233-0343 (top), HE 0945-1435 (middle) and HD $140283([\mathrm{Fe} / \mathrm{H}] \sim-2.4$; bottom). Stars are shown offset vertically for clarity.

enough that even after the refinement, HE 0233-0343 should remain in the metallicity gap. This is a very important result as at this time we are aware of only one other star observed in that metallicity range, HE 0557-4840. The spectra of HE 0233-0343 need further analysis also because of the existence of weak broad features close to the Fe I lines. From preliminary measurements, we believe these may arise from broad $\mathrm{Fe}$ I absorptions, blue shifted by $0.09 \mathrm{~nm}$ relative to the weak line spectrum. This star also presents a weak $G$ band of $\mathrm{CH}$ that will be analysed in order to get a proper carbon abundance, which in turn should be used in the spectral analysis.

\section{Lithium}

The other important outcome was the detection of the neutral lithium resonance line $\mathrm{Li} \mathrm{I} 670.8 \mathrm{~nm}$ in the spectra of these two stars. This decreases the lower metallicity limit for the detection of this element, from $[\mathrm{Fe} / \mathrm{H}] \sim-3.80$ down to $[\mathrm{Fe} / \mathrm{H}] \sim-4.25$. The former value corresponds approximately to the minimum metallicity value for which the ESO Large Programme 'First Stars' has reported lithium abundances (Bonifacio et al 2007) and the last value comes from table 1 in our paper. However, the detection at $[\mathrm{Fe} / \mathrm{H}] \sim$ -3.80 corresponds to a cool subgiant, the lithium of which is expected to be depleted; indeed, it is observed to be very low. In theory, our two observed stars should be better indicators of the primordial lithium content as they are either dwarfs or warm subgiants. Figure 3 shows the good quality of our observations in the wavelength range of the lithium resonance line and how weak that line is in the spectra of the two stars, having equivalent widths of $0.8 \mathrm{pm}$ (HE 0233-0343) and $1.0 \mathrm{pm}$ (HE 0945-1345).

The possible range in stellar parameters is too large to allow an accurate abundance determination for $\mathrm{Li}$, with $1 \mathrm{D}$ LTE $A(\mathrm{Li})$ in the range $1.90-1.70$ for HE $0233-0343$ and 1.99-1.80 for HE $0945-1345$. A recent study for lithium abundance in extremely metal-poor stars (Bonifacio et al 2007) suggests that stars with lower metallicities have lower lithium content. Our values seem to be lower than typical LTE

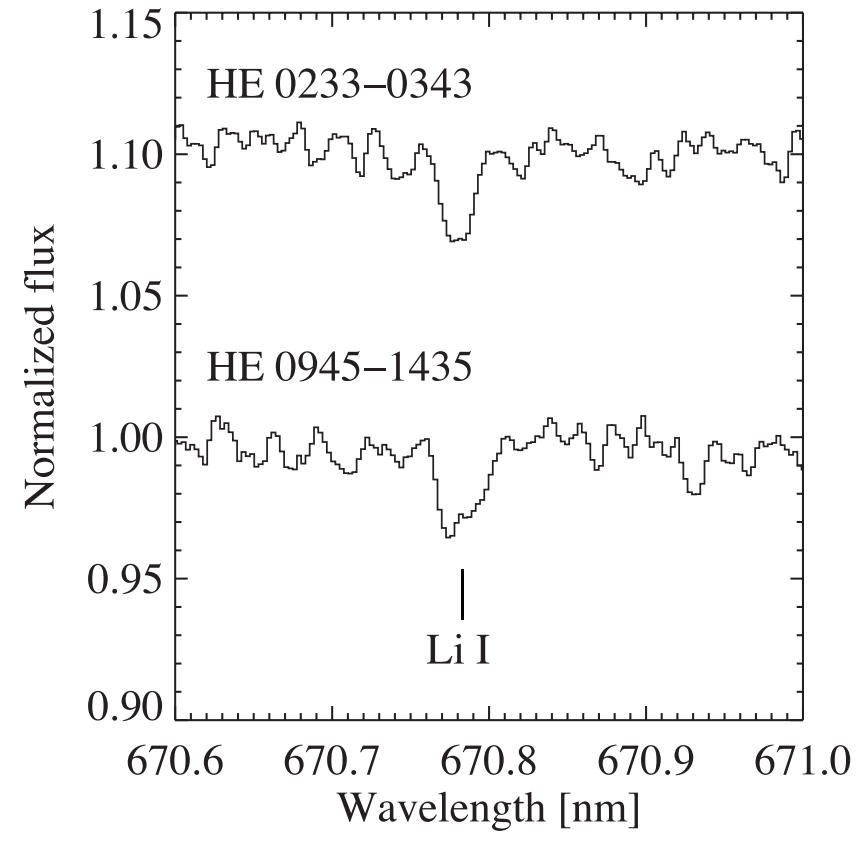

Figure 3. VLT/UVES spectra of the Li I $670.8 \mathrm{~nm}$ line for HE 0233-0343 and HE 0945-1435. Stars are shown offset vertically for clarity.

values for stars with $[\mathrm{Fe} / \mathrm{H}] \sim-2.50$ but similar to the values for stars with $[\mathrm{Fe} / \mathrm{H}] \sim-3.30$.

\section{Conclusions}

Spectra for a sample of 30 HES stars were acquired at the VLT and are being analysed. According to the spectral analysis of the observed hydrogen and Fe I lines, we have two dwarf/subgiant stars with very low metallicities $([\mathrm{Fe} / \mathrm{H}] \sim-4.0)$ and lithium detections. These detections are a big achievement because of the extremely low metallicity observed, the lowest metallicity for detections in dwarf or warm subgiant stars at this time. However, these metallicity results are very preliminary and they need to be confirmed by a more thorough analysis. 


\section{Acknowledgments}

We would like to congratulate the conference organizers for a very stimulating symposium and wish happy birthday to BenGt. AEGP has been supported by a research grant from the Science and Technology Facilities Council (UK) and an International Joint Project funded by the Royal Society (UK). NC acknowledges financial support by the Knut and Alice Wallenberg Foundation and by Deutsche Forschungsgemeinschaft under grant Ch 214/3-2.

\section{Appendix. Discussion}

Q: (Poul Erik Nissen) Do you determine $[\mathrm{Fe} / \mathrm{H}]$ from $\mathrm{Fe}$ I lines? Fe II lines may give higher values of $[\mathrm{Fe} / \mathrm{H}]$ by up to 0.2 dex due to non-LTE effects.

A: Yes, non-LTE studies of Fe I lines suggest they are affected by departures of LTE, therefore higher metallicities are expected. We are looking at the moment at possible $\mathrm{Fe}$ II lines that are in the limits of detection. I should add that the $\mathrm{Fe}$ II lines that are included in the line list have to be explored.

Q: (Ann Boesgaard) We are used to seeing a straight-line plateau in $\mathrm{A}(\mathrm{Li})$ versus $[\mathrm{Fe} / \mathrm{H}]$ yet you now show a decreasing relation-lower $\mathrm{Fe}$ and lower Li. What about a plateau and, at lower Fe, decreasing Li?

A: That would be an interesting finding and a challenge for theoreticians to explain. There are suggestions in Bonifacio et al (2008) that the Li-versus-Fe for Asplund et al (2006) seems to be a Li-versus-Teff trend. However, the data of Bonifacio does not seem to show that. We should check which is the case for our sample once better Teff are determined.

Q: (Jeff Linsky) At present the best tests of conditions during Big Bang Nucleosynthesis are the deuterium and WMAP results. The measured lithium abundances for low metallicity stars appear to be inconsistent. However, the ${ }^{4} \mathrm{He}$ results are also inconsistent with the deuterium and WMAP results. It is very important to understand why both lithium and ${ }^{4} \mathrm{He}$ are inconsistent. My guess is that the solution to the lithium inconsistency will also explain the ${ }^{4} \mathrm{He}$ inconsistency.

A: I answer with a question: can the determinations from the WMAP for the baryon density be affected by any systematic errors?

Q: (Andreas Korn) It is true that diffusion complicates the picture. However, atomic diffusion is a fundamental physical process and as such 'inevitable'. To which extent it affects Li abundances of metal-poor stars is under current investigation. But it's important to realize the ${ }^{6} \mathrm{Li}$ depletions do not have to be more severe than ${ }^{7} \mathrm{Li}$ depletions due to diffusion (disregarding pre-MS depletion which in itself is rather uncertain).

Q: (Martin Asplund) Just a quick follow-on to Andreas' comment on ${ }^{6} \mathrm{Li}$ and ${ }^{7} \mathrm{Li}$ depletion: it is true that ${ }^{6} \mathrm{Li}$ depletion does not automatically have to be higher than the corresponding ${ }^{7} \mathrm{Li}$ depletion, since it depends on the mixing time-scales. For rotationally-induced mixing ${ }^{6} \mathrm{Li}$ depletion is always significantly higher than for ${ }^{7} \mathrm{Li}$. In the case of diffusion plus turbulent mixing the two depletions are only similar for modest depletion factors $(\sim 0.2$ dex $)$ while ${ }^{6} \mathrm{Li}$ depletion becomes much more severe for larger ${ }^{7} \mathrm{Li}$ depletion factors. Finally, during pre-MS the ${ }^{6} \mathrm{Li}$ depletion is predicted to be substantial (roughly $\geqslant 0.3 \mathrm{dex}$ ) while little or no ${ }^{7} \mathrm{Li}$ depletion is expected.

Q: (Hans-Guenter Ludwig) Comment: First, I would like to congratulate you for finding the most overall metal-poor dwarf so far. Second, I think one should not talk about a metallicity-gap between the bulk of metal-poor stars and the Christlieb-Frebel-Norris objects since they might have formed under different conditions.

\section{References}

Barklem P S, Stempels H C, Allende Prieto C, Kochukhov O P, Piskunov N and O'Mara B J 2002 Astron. Astrophys. 385 951-67

Beers T C and Christlieb N 2005 Annu. Rev. Astron. Astrophys. 43 531-80

Bonifacio P et al 2007 Astron. Astrophys. 462 851-64

Christlieb N, Bessell M S, Beers T C, Gustafsson B, Korn A, Barklem P S, Karlsson T, Mizuno-Wiedner M and Rossi S 2002 Nature 419 904-6

Christlieb N, Schörck T, Frebel A, Beers T, Wisotzki L and Reimers D 2008 Astron. Astrophys. 484 721-32

Collet R, Asplund M and Thévenin F 2005 Astron. Astrophys. 442 $643-50$

Demarque P, Woo J H, Kim Y C and Yi S K 2004 Astrophys. J. Suppl. 155 667-74

Frebel A et al 2005 Nature 434 871-3

Gustafsson B, Edvardsson B, Eriksson K, Jørgensen U G, Nordlund $\AA$ and Plez B 2008 Astron. Astrophys. 486 951-70

Karlsson T 2006 Astrophys. J. Lett. 641 L41-4

Norris J E, Christlieb N, Korn A J, Eriksson K, Bessell M S, Beers T C, Wisotzki L and Reimers D 2007 Astrophys. J. 670 $774-88$

Tsuribe T and Omukai K 2008 Astrophys. J. Lett. 676 L45-8 